\title{
Angular Correlations in Coherent Electron Nanodiffraction of a Metallic Glass
}

\author{
Jinwoo Hwang $^{*}$, Y. E. Kalay ${ }^{* *}{ }^{* * *}$, M. J. Kramer ${ }^{* * *}$, and P. M. Voyles ${ }^{*}$ \\ ${ }^{*}$ Dept. of Materials Science, University of Wisconsin-Madison, Madison, WI, United States. \\ ** Dept. of Metallurgical and Materials Engineering, Middle East Technical University, Ankara, \\ Turkey. \\ ${ }^{* * *}$ Dept. of Materials Science and Engineering, Ames National Laboratory, Ames, IA, United \\ States.
}

We examined nanometer scale structure in Zr-based bulk metallic glass (BMG) using coherent electron nanodiffraction [1]. Spatial fluctuations in the diffracted intensity, measured in fluctuation electron microscopy (FEM), show that there is a structural heterogeneity at the $1-2 \mathrm{~nm}$ scale [1]. Each nanodiffraction pattern, such as Figure 1(a), shows Bragg diffraction speckles from local medium range order (MRO), so the rotational symmetry of the speckles as a function of scattering vector magnitude, $k$, should reveal the internal structure of the MRO. However, identifying the important symmetry in nanodiffraction is a difficult task, because the TEM sample may contain several ordered regions through the thickness whose speckles may overlap, and the important speckles may be hidden by scattering from statistically insignificant structures.

The angular correlation function, $C_{k}(\Delta)$, can reveal this structural information $[2,3]$.

$$
C_{k}(\Delta)=\frac{\langle I(k, \varphi) I(k, \varphi+\Delta)\rangle_{\varphi}-\langle I(k, \varphi)\rangle_{\varphi}^{2}}{\langle I(k, \varphi)\rangle_{\varphi}^{2}}
$$

where $I(k, \varphi)$ is the diffracted intensity in polar coordinates. $C_{k}(\Delta)$ shows the correlations between two intensities at the same $k$, separated by angle $\Delta$, averaged over the entire angle $\varphi$. Figure 1(b) shows the unwrapped $I(k, \varphi)$ at $k=3.93 \mathrm{~nm}^{-1}$ from the diffraction pattern in Figure 1(a). Figure 1(c) is the corresponding $C(\Delta)$ which shows strong 6 fold symmetry. We further performed power spectrum $\left(P_{n}\right)$ analysis to quantitatively extract the symmetry in $C_{k}(\Delta)$. The $P_{n}$ of $C_{k}(\Delta)$, shown in Figure 1(d), has even greater sensitivity to the rotational symmetry. In this case, it has a single strong peak at $n=6$.

We calculated the average of $P_{n}(k)$ over 2,077 nanodiffraction patterns from as-quenched $\mathrm{Zr}_{50} \mathrm{Cu}_{45} \mathrm{Al}_{5}$ BMG ribbons. Figure 2 (a) shows the average $P_{n}(k)$ for $n=2$ to 10 . Near the $1^{\text {st }}$ diffraction ring $\left(k \sim 4.0 \mathrm{~nm}^{-1}\right), n=2$ (Figure 2(b)) is higher than any other $n$, so Friedel symmetry is the dominant feature. $n=4$ and 6 , which are signatures of crystalline order, are also relatively strong at similar $k$, but have several peaks in $k$, especially $n=6$. This suggests that there may be different types of crystalline-like MRO with different interatomic spacings, which is consistent to our findings using FEM [1]. The shape of $P_{n}(k)$ is different for $n=2,4$, and 6 , so the $n=4$ and 6 data are not simple results of random combinations of $n=2$.

We also observe a peak for $n=10$ in Figure 2(b). $n=10$ fold is a 5-fold symmetry doubled by Friedel conjugates, so it may arise from icosahedral-like atomic clusters often identified in molecular dynamics simulations [5]. However, nanodiffraction simulations from those widelyaccepted models do not agree with crystalline-like MRO. Additional modeling results will be discussed [6]. 


\section{References}

[1] Jinwoo Hwang and P. M. Voyles, Microscopy and Microanalysis 17 (2011) 67-74.

[2] Wochner et al., PNAS 106, (2009) 11511.

[3] J. M. Gibson, M. M. J. Treacy, T. Sun, and N. J. Zaluzec Phys. Rev. Lett. 105 (2010) 125504.

[4] M. Altarelli, R. P. Kurta, and I. A. Vartanyants, Phys. Rev. B 82 (2010) 104207.

[5] H. W. Sheng, W. K. Luo, F. M. Alamgir, J. M. Bai, and E. Ma, Nature 439 (2006) 26.

[6] This work is supported by the National Science Foundation (CMMI- 0824719).

(a)

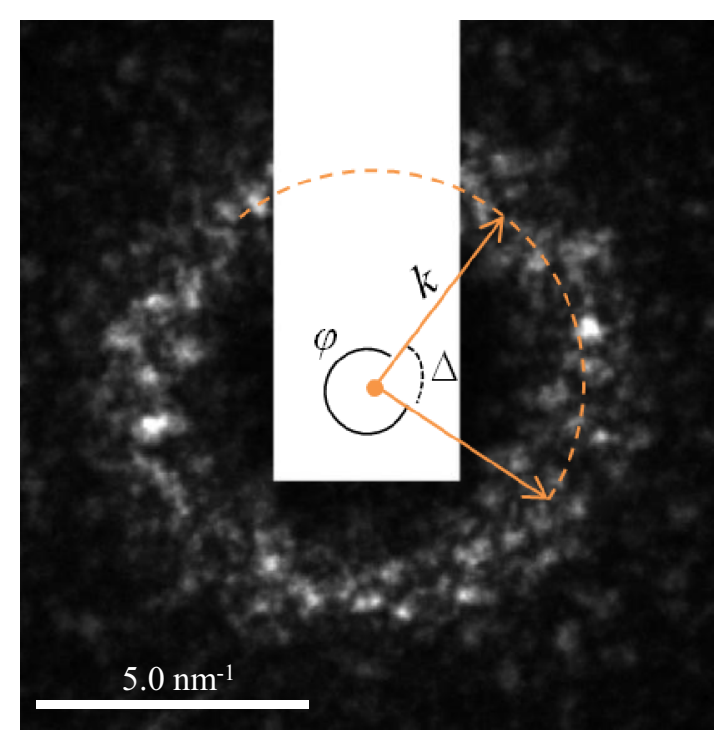

(b)

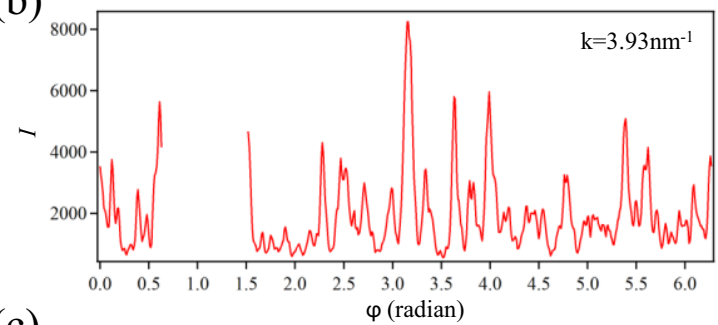

(c)

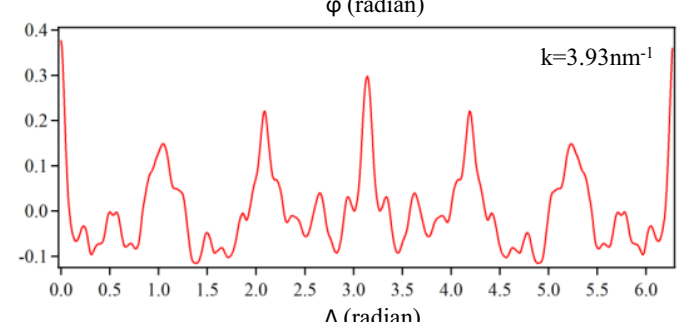

(d)

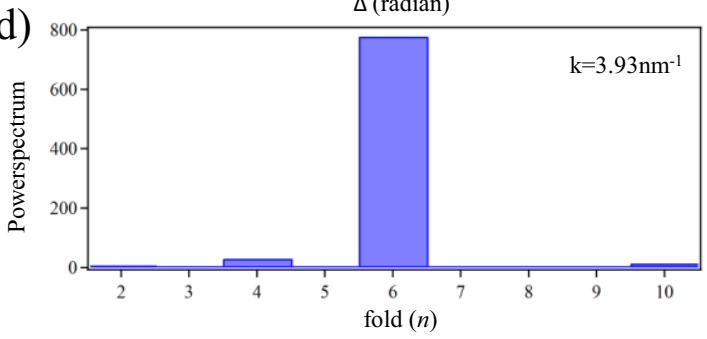

Figure 1. (a) Example of a nanodiffraction acquired with $2 \mathrm{~nm}$ diameter probe. (b) $I(\varphi)$, (c) $C(\Delta)$, and (d) $P_{n}$ at $k=3.93 \mathrm{~nm}^{-1}$ of the pattern in (a).
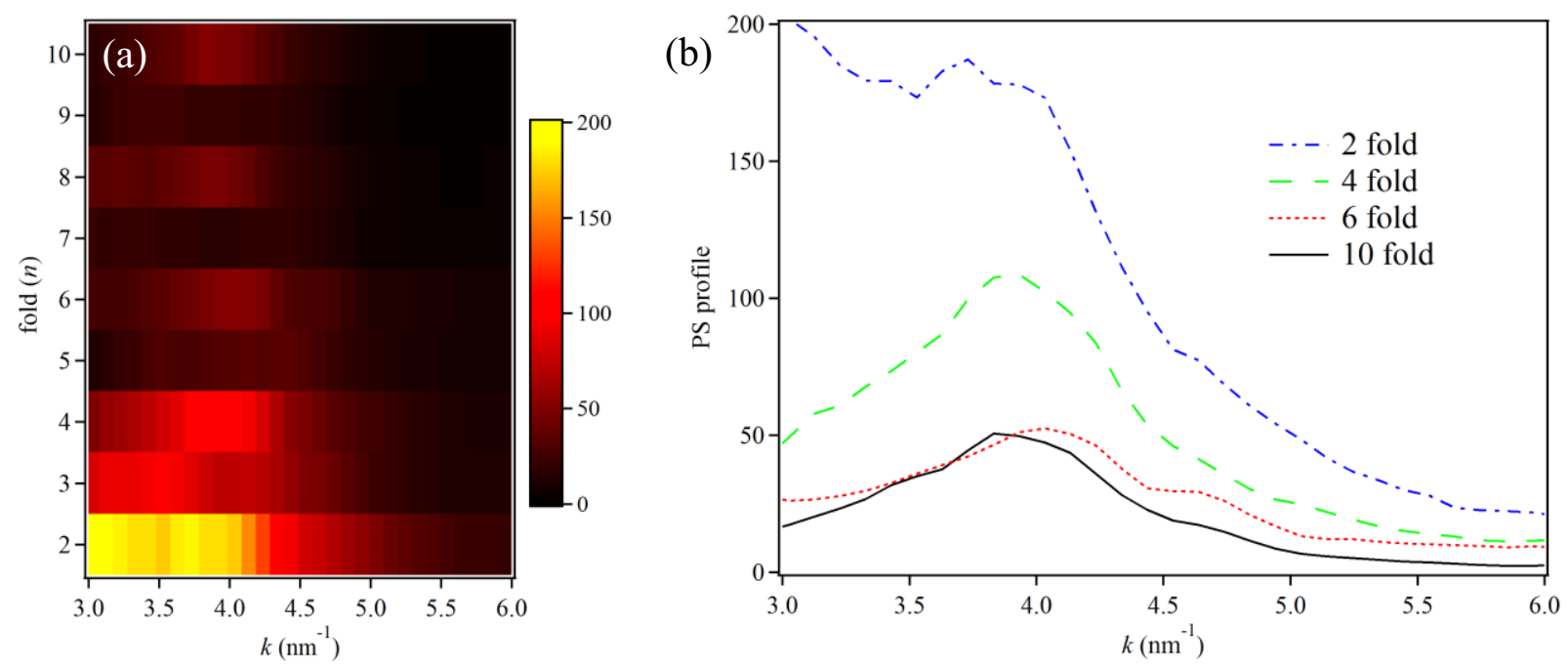

Figure 2. (a) Average $P_{n}(k)$, and (b) line profiles at $n=2,4,6$, and 10 . 